\title{
Bilingüismo individual en escuelas de educación indígenas
}

\author{
Eva América Mayagoitia Padilla \\ Profesora investigadora \\ Universidad Pedagógica Nacional del Estado de Chihuahua \\ campus Chihuahua
}

\section{Resumen}

ste artículo reporta los resultados $\checkmark$ de un estudio efectuado en dos escuelas de educación indígena del estado de Chihuahua, México. Se analiza el bilingüismo individual que practican alumnos de primer ciclo de primaria, identificando los grupos lingüísticos existentes. Se utilizó el enfoque de métodos mixtos, desde un diseño incrustado con predominio del componente cualitativo, efectuando procesos de observación del comportamiento lingüístico de los niños(as), y la aplicación de entrevistas semiestructuradas con padres y alumnos. Se identificaron cuatro tipos de bilingüismo individual, en cuya conformación influyen los usos lingüísticos producidos en las familias, destacando la decisión de los padres de enseñar a sus hijos(as), una, otra u ambas lenguas como lengua(s) materna(s). Sobresalió la influencia de la edad y la experiencia escolar en el aprendizaje del castellano como segunda lengua y la condición irreversible del uso del castellano como única lengua, en los alumnos monolingües en castellano.

Palabras clave: bilingüismo individual, grupos lingüísticos, escuelas indígenas.

\section{Introducción}

En este trabajo se exponen los resultados de un estudio que analizó el bilingüismo individual de 32 niños(as) rarámuris, inscritos en primer año de primaria en dos escuelas indígenas ubicadas en las comunidades de San Elías (SE) y San Ignacio (SI) de Arareco en el municipio de Bocoyna, Chihuahua, México.

Forma parte de una investigación más amplia, que analizó las diferencias en el aprendizaje del sistema de escritura, entre grupos lingüísticos. De ahí, la necesidad de reconocer de manera previa, el bilingüismo individual de los niños(as), identificando así mismo, los grupos lingüísticos 
existentes. Las preguntas que guiaron el estudio fueron: ¿Qué uso tiene el rarámuri y el castellano en el contexto familiar de los alumnos? ¿Cuáles son las competencias comunicativas de los padres en ambas lenguas? ¿Cuáles son las competencias comunicativas en la L1 y L2 de los alumnos? y derivado de ello ¿Qué tipo de bilingüismo practican? y ¿Cuáles son los grupos lingüísticos existentes?

\section{Objetivos}

1. Valorar la competencia comunicativa de los padres, identificando su lengua materna, así como su competencia comunicativa en rarámuri y castellano.

2. Analizar el tipo de comunicación lingüística que se establece al interior de las familias.

3. Evaluar la competencia comunicativa de los niños, identificando su lengua materna, así como su competencia comunicativa en ambas lenguas.

4. Identificar el bilingüismo individual y los subgrupos lingüísticos que prevalecen.

\section{Método}

Se eligió el enfoque de Métodos Mixtos que permite la obtención y el análisis de datos cualitativos y cuantitativos en un mismo estudio (Creswell y Plano Clark, 2007), lo que supuso efectuar observaciones sistemáticas sobre el comportamiento lingüístico de cada niño en el grupo y la escuela, complementando la información con entrevistas semiestructuradas que se trabajaron con los alumnos y sus padres. Con los insumos obtenidos, se construyó un registro personal de cada alumno, cuyo análisis permitió la identificación de su bilingüismo individual y gracias al apoyo de un programa estadístico fue posible identificar también los grupos lingüísticos prevalecientes.

\section{Fundamentos teóricos}

A diferencia del bilingüismo social, que analiza situaciones derivadas del contacto entre lenguas, el bilingüismo individual se refiere al cambio de código o elección de una lengua por individuos concretos. Existen dos posturas para definir a una persona bilingüe: a) las que afirman que el bilingüe es similar a la suma de dos monolingües, lo que implica ser homogéneamente competente en el uso de ambas lenguas y b) las que sostienen que bilingüe es la persona que añade cualquier grado de conocimiento de una nueva lengua, al que tiene sobre la propia, lo que supone ser heterogéneamente competente en su uso (Vigil, 2008).

Esta autora plantea que las personas bilingües lo son, porque las necesidades comunicativas de su lengua materna se amplían hacia una segunda lengua, siendo heterogéneas en cada persona por situaciones sociales, psicológicas y lingüísticas. Añade que sociolingüistas connotados, han demostrado que en comunidades con prevalencia de bilingüismo social, las lenguas en contacto se "reparten" con problemas las tareas comunicativas, que en un grupo monolingüe serían cubiertas por una sola lengua, afectando, el desarrollo académicocognitivo y social de los hablantes.

Desde tales condiciones, la educación en contextos bilingües, debe generar las 
condiciones propiciatorias para un bilingüismo aditivo, reconociendo que éste y el desarrollo cognitivo están estrechamente relacionados y que el aprendizaje competente en dos idiomas, da como resultado un incremento sustancial en la flexibilidad mental, la formación de conceptos y la diversificación de habilidades mentales (López, 2004). La importancia de valorar el bilingüismo individual en comunidades bilingües, radica en que proporciona una plataforma comprensiva de la realidad lingüística de cada niño y del grupo escolar, posibilitando la planificación de las tareas educativas, con calidad y pertinencia lingüística.

En este caso, previamente se eligió una tipología que surgió de la conjugación de las propuestas de Coronado (1996) y López (1989), cuyos bilingüismos se clasificaron como:

1.- Monolingüe en rarámuri, que se refiere a los alumnos que en los procesos comunicativos usan predominantemente la lengua indígena.

2.- Bilingüe con predominio del rarámuri, característica de los estudiantes que en los procesos comunicativos usan el rarámuri y el castellano pero con mucho mayor predominio y presencia de la lengua de grupo.

3.- Bilingüe funcional que refiere a los individuos que utilizan ambas lenguas en la vida cotidiana, distinguiendo entre funciones, ámbitos y contextos de uso, es decir, no usan las lenguas equilibradamente, sino que cada una se utiliza en condiciones de diferenciación y jerar- quización social, implicando un manejo suficiente de ambas.

3.- Bilingüe con predominio del castellano que caracteriza a los alumnos que en la comunicación utilizan las dos lenguas con una mayor presencia y dominio del castellano.

4.- Monolingüe en castellano que refiere a niños(as) que en los eventos comunicativos usan predominantemente el castellano.

La tipología sirvió de base para el estudio empírico, cuyos resultados se describen a continuación.

\section{Resultados}

\section{Tipo de comunidades bilingües}

Hablar dos lenguas en un espacio social determinado, hace posible distintos tipos de sociedades bilingües que son: a) comunidades tipo 1 donde un grupo de personas habla únicamente la lengua $\mathrm{X}$, $\mathrm{y}$ otro solo habla la lengua $\mathrm{Y}, \mathrm{b}$ ) comunidades tipo 2 donde un gran grupo de individuos hablan ambas lenguas y muy pocos son monolingües en una u otra lengua, y c) comunidades tipo 3 donde coexisten: un grupo monolingüe en la lengua $\mathrm{X}$, un grupo bilingüe en $\mathrm{X}$ y $\mathrm{Y}$ y otro grupo monolingüe en $\mathrm{Y}$ (Appel y Muysken,1996).

Las comunidades estudiadas pertenecen al tipo 3, pues desde las primeras observaciones se identificó una minoría de personas monolingües en lengua rarámuri o monolingües en castellano y una gran variedad de personas bilingües que se comunicaban en una, otra $u$ ambas lenguas, 
con distintas intensidades y matices, para distintas funciones y necesidades sociales.

\section{Tipos de bilingüismo en la realidad}

Los resultados mostraron la inexistencia de alumnos "bilingües con predominio del castellano", evidenciando el prestigio de ésta lengua y el desplazamiento paulatino de la lengua indígena que se refleja en el desarrollo de un bilingüismo sustractivo de la lengua de grupo (Hirsch, González y Cicconé, 2006). Se acentuó en los alumnos cuya lengua materna es el castellano, pues se esperaba que además de su dominio, manifestaran también, ciertas competencias en lengua indígena. Lamentablemente, tal suposición resultó equivocada.

Se encontraron cuatro tipos de bilingüismo que evidencian la interrelación entre condiciones sociolingüísticas y tipos de bilingüismo individual. Así, los niños monolingües en lengua indígena (seis casos), mayoritariamente (cuatro) viven en SE. Contrariamente, los monolingües en español (siete casos) en su mayoría (seis) viven en SI. Los bilingües con predominio del rarámuri (10) se distribuyeron equitativamente (cinco en SI y cinco en SE y los bilingües funcionales (nueve casos) mayoritariamente (siete) son de SI.

En síntesis, en SE, el prestigio social del rarámuri resulta evidente cuando predomina el monolingüismo en rarámuri y el bilingüismo con predominio de esta lengua. Por el contrario, resultó representativo del desplazamiento paulatino de la lengua de grupo evidenciada en una mayor diversidad de bilingüismos, entre ellos: el bilingüismo funcional, el monolingüismo en castellano y en menor medida, el bilingüismo con predominio del rarámuri. Coincidentemente, en términos globales el predominio de alumnos bilingües alcanzó al $59.3 \%$ del total de la población, mientras los monolingües en castellano alcanzaron el 21.8\% y los monolingües en lengua indígena sumaron el $18.7 \%$.

\section{Características de los grupos lingüísticos}

Los datos recabados posibilitaron la siguiente caracterización:

1.- Monolingües en rarámuri. Este subgrupo que mayoritariamente vive en SE incluyó un total de seis alumnos: cinco niñas y un niño que cursan primer grado de primaria. De ellos solo cuatro cursaron preescolar, mientras que dos no tenían antecedentes escolares. Sus edades fluctuaron entre cinco y siete años, predominando los de seis años (cuatro casos). Todos se comunican muy bien en rarámuri, que es su lengua materna y la que cubre la mayoría de las funciones sociales que realizan. Por el contrario, su dominio del castellano osciló desde no hablar ni comprender nada (cuatro casos) a hablar algunas palabras y entender instrucciones muy sencillas (dos).

Los padres entrevistados (cinco mujeres y un varón), así como sus cónyuges nacieron en la comunidad y su lengua materna es el rarámuri, que utilizan prioritariamente en la comunicación intrafamiliar. El 83\% habla castellano con fluidez, mientras que el $16.7 \%$ lo hace incipientemente. Solo dos leen y escriben con dificultad en ambas lenguas (33.3\%), 
mientras que el $66.7 \%$ declararon no saber. Dos de ellos nunca hablan castellano con sus hijos, uno casi nunca, dos más ocasionalmente y solo uno utiliza ambas lenguas en la comunicación con los hijos.

2.- Bilingüe con predominio del rarámuri. Incluyó un total de 10 alumnos, seis mujeres y cuatro varones, que viven equitativamente en ambas comunidades (cinco en SE y cinco en SI). Solo cinco cursaron preescolar y se caracterizaron por su diversidad en cuanto al grado escolar (seis en 1 er grado y cuatro en $2^{\circ}$ ) así como por los rangos de edad que oscilaron entre seis y 12 años. Fueron los alumnos con más años de permanencia en la escuela.

Todos se comunican perfectamente en rarámuri, que es su lengua materna, siendo también, la que cubre la mayoría de las funciones sociales que realizan. En castellano son capaces de identificar objetos cotidianos sin dificultad y ejecutan instrucciones verbales que implican varias acciones simultáneamente (dos o tres). Valorar su competencia expresiva resultó muy difícil, es decir, este subgrupo sobresale por sus habilidades incipientes para entender castellano mas no para hablarlo.

Los padres entrevistados (cinco varones y cinco mujeres) tienen características similares a los del subgrupo de monolingües en lengua indígena. Todos nacieron en la comunidad, al igual que sus cónyuges y en su totalidad la lengua materna es el rarámuri cuyo dominio oral es alto. La gran mayoría (nueve) declararon usar frecuentemente la lengua in- dígena en la comunicación con los hijos, mientras que solo uno declaró que casi nunca. Esta situación en el caso del hijo, se compensa gracias a que la madre se comunica con él siempre en rarámuri.

En relación al castellano, siete lo hablan y entienden perfectamente y los tres restantes lo hacen incipientemente, solo cinco leen y escriben con dificultad en ambas lenguas, mientras que los cinco restantes son analfabetos. Dos padres declararon que en la comunicación intrafamiliar siempre lo usan, cinco en ocasiones, dos casi nunca y solo uno nunca.

3.- Bilingüe funcional. Esta categoría incluyó nueve alumnos que mayoritariamente (siete) viven en SI, donde aprenden castellano a edades tempranas, pues auxilian a sus madres en la venta de artesanías. El 50\% cursó preescolar y se caracterizaron por su diversidad de rangos de edad (uno de 5 años, dos de 6 , tres de 7, dos de 8 y uno de 12). Mayoritariamente pertenecen a primer grado (ocho) y solo un caso a segundo grado.

Fueron el grupo con mayores competencias lingüísticas en ambas lenguas. Todos señalaron al rarámuri como lengua materna, pero en tres casos se presupone la presencia de un bilingüismo de cuna en el que las dos lenguas fueron aprendidas simultáneamente. En los seis restantes, se corroboró la presencia de un bilingüismo consecutivo temprano, donde el rarámuri fue la primera lengua y se acompañó desde edades tempranas del aprendizaje incipiente del castellano. Así, no se trata de un bilingüismo equi- 
librado, sino de un bilingüismo funcional en el que la lengua indígena cubre la mayoría de las necesidades sociales, mientras que el español se usa para desarrollar funciones jerárquicamente diferenciadas (López, 1989). Así, todos relatan hechos sencillos con claridad y adicionalmente, cinco de ellos toman iniciativa, siguiendo una secuencia lógica y coherente al conversar en castellano.

Sus competencias bilingües coinciden con las características lingüísticas de los padres entrevistados: cinco hombres y cuatro mujeres, ocho de los cuales hablan perfectamente en rarámuri, mientras que solo un varón lo hace incipientemente. Sobresale que tres de las madres son monolingües en rarámuri lo que explica la simultaneidad del uso de las lenguas al interior de los hogares. El 88.9\% la usan permanentemente para comunicarse con los hijos, mientras que solo uno la utiliza incipientemente.

Sus competencias orales en castellano también son altas en el $88.9 \%$ de los casos (ocho) mientras solo un caso manifestó hablarlo poco. El $44.4 \%$ lee y escribe muy bien, $33.3 \%$ poco y $22.2 \%$ son analfabetos. Sobresale la diversidad de condiciones de uso en la comunicación intrafamiliar: solo el $22.2 \%$ lo usa mucho, el $55.5 \%$ a veces y el $22.2 \%$ nunca.

4.- Monolingüe en castellano. Contrario a sus pares monolingües en rarámuri, los siete estudiantes (cinco varones y dos mujeres) viven mayoritariamente en SI. Todos cursaban primer grado de primaria, contando mayoritariamente (seis) con antecedentes de preescolar. Sus edades oscilaron entre seis y siete años. Sus competencias en rarámuri se limitan a entender algunas palabras sin lograr expresarlas de manera verbal. Su lengua materna es el castellano y se diferencian de los otros subgrupos por el peso que en los procesos de construcción de conocimientos tiene el lenguaje oral, como mecanismo fundamental del pensamiento y la acción. Así, relatan hechos con claridad e hilaridad y son capaces de mantener conversaciones sobre tópicos comunes, siguiendo una secuencia lógica y coherente.

A la entrevista asistieron siete madres, seis de las cuales nacieron en una comunidad rarámuri. La lengua materna de la mayoría (seis) es el castellano y reconocieron no saber rarámuri a pesar de sus costumbres indígenas. Así, solo en uno de los hogares se habla rarámuri pero de manera incipiente porque el padre lo prohíbe, mientras que en los seis restantes la única lengua de comunicación en el hogar es el castellano, ya que cuatro hogares tienen jefatura femenina y en los restantes, el padre también es monolingüe en castellano. Sin embargo, solo tres saben leer y escribir, tres lo hacen incipientemente y otra es analfabeta.

\section{Conclusiones}

Los resultados evidenciaron cuatro tipos de bilingüismo individual que se producen fundamentalmente por:

1.- Los usos lingüísticos al interior de los hogares, destacando la influencia de los 
padres en el desarrollo lingüístico de los niños(as), dada su función de primeros educadores. Son ellos quienes en virtud de sus condiciones económicas, sociopolíticas y culturales, optan por la enseñanza de una, otra y en contadas ocasiones ambas lenguas como lengua(s) materna(s), decisión en la que se juega la continuidad, pero también la interrupción del vínculo intergeneracional representado por la lengua indígena (Hirsch, González y Cicconé, 2006).

2.- Cuando se opta por la enseñanza del rarámuri como lengua materna, se produce un bilingüismo individual de carácter consecutivo (López, 1989), donde el monolingüismo en rarámuri, es generalmente una condición transitoria, que deriva luego, en bilingüismo con predominio del rarámuri y/o en bilingüismo funcional. Así, el nivel de dominio del castellano depende de la edad en que se inicia su aprendizaje como segunda lengua y de la experiencia escolar, evidenciando la influencia de la escuela en los procesos de castellanización (López, 2004).

3.- Cuando se opta por la enseñanza del castellano como lengua materna, el monolingüismo en castellano se convierte en una condición lingüística irreversible, que evidencia un fenómeno creciente en las comunidades indígenas del estado. El surgimiento de identidades híbridas se distancian de las formas tradicionales de conceptualizar lo indígena, pues ser hablante de la lengua de grupo, no es ya una condición fundamental para considerarse como tal.
4.- Se generan nuevas realidades que obligan a repensar lo étnico, así como las políticas educativas para la enseñanza de lenguas en las escuelas indígenas, desde la diversidad lingüística que prevalece en las escuelas, implicando también, la implementación de estrategias lingüísticas diversas, que en el caso del aprendizaje del sistema de escritura supone: promover simultáneamente el proceso de lectoescritura en lengua indígena y también en castellano, dependiendo de cuál es la lengua materna de cada niño(a), lo que sin duda, hace más compleja la ya complejísima realidad educativa de las poblaciones originarias del estado.

\section{Referencias}

Appel, R. y Muysken, P. (1996). Introducción: Bilingüismo y contacto entre lenguas en Bilingüismo y contacto entre lenguas. Arial lingüística. Barcelona.

Coronado, G. (1996). El bilingüismo como alternativa frente a la diversidad lingüística y cultural. En. Muñoz, H., Lewin, F., y Gumperz, J. (Eds.) El significado de la diversidad lingüística y cultural. (pp. 64-73). UAM Iztapalapa INAH. México.

Creswell, J. y Plano Clarck, V. (2007). Designing and conducting Mixed Methods Research. Thousand Oaks. Sage Publications. USA.

Hirsch, S., González H. y Ciccone, F. (2006). Lengua e identidad: ideologías lingüísticas, pérdida y revitalización de la lengua entre los tapietes. Recuperado 9 de febrero de 2011 en: http://www.iai.spk-berlin.de/ fileadmin/dokumentenbibliothek/Indiana/Indiana_ 23/08HirschGonzalezCiccone_neu.pdf

López, L. (1989). La naturaleza del lenguaje. Lengua 2. UNESCO/ORELAC. Santiago de Chile.

López, L. (2003). ¿Dónde estamos con la enseñanza del castellano como segunda lengua en América Latina? En Jung, I y López, L. (Comps). Abriendo la escuela. Lingüística aplicada a la enseñanza de lenguas. pp.39-71. Ediciones Morata. Madrid.

Vigil, N. (2008) Bilingüismo, individuo y sociedad. Recuperado el 12 de julio de 2012 en: http://nilavigil. wordpress.com/2008/06/12/bilinguismo-individuoy-sociedad/. 
